Mclntyre et al. Molecular markers of stormwater runoff and bioretention treatment

\title{
Confirmation of stormwater bioretention treatment effectiveness using molecular indicators of cardiovascular toxicity in developing fish
}

Jenifer K. McIntyre ${ }^{*^{1 \wedge}}$, Richard C. Edmunds ${ }^{2 \wedge}$, Maria G. Redig ${ }^{3}$, Emma M. Mudrock ${ }^{1}$, Jay W. Davis $^{4}$, John P. Incardona ${ }^{5}$, John D. Stark ${ }^{1}$, Nathaniel L. Scholz $^{5}$

${ }^{1}$ Washington State University, Puyallup Research and Extension Center, 2606 W. Pioneer Ave., Puyallup, WA 98371

${ }^{2}$ National Research Associateship Program, Northwest Fisheries Science Center, National Marine Fisheries Service, NOAA, 2725 Montlake Blvd. E., Seattle, WA 98112

${ }^{3}$ Evergreen State College, 2700 Parkway NW, Olympia, WA 98505

${ }^{4}$ U.S. Fish and Wildlife Service, Washington Fish and Wildlife Office, 510 Desmond Dr. S.E., Lacey, WA 98503

${ }^{5}$ Environmental and Fisheries Science Division, Northwest Fisheries Science Center, National Marine Fisheries Service, NOAA, 2725 Montlake Blvd. E., Seattle, WA 98112

\section{Supporting Information}

Table S1: Analytical chemistry for runoff samples and embryo medium Table S2: PAH measurements in unfiltered runoff and filtered runoff Table S3: Quantitative PCR primer information Table S4: Gene expression of molecular biomarkers for runoff from three storms Table S5: Gene expression from filtered runoff or clean leach water Figure S1: Cardiac morphometrics and $n p p b$ induction for dilutions of unfiltered May and Sep runoff

Figure S2: Immunofluoresence of CYP1A from exposure to unfiltered and filtered Sep runoff 
Mclntyre et al. Molecular markers of stormwater runoff and bioretention treatment

Table S1. Analytical chemistry for runoff samples and embryo medium used in zebrafish exposures. Metals are dissolved $(<0.45 \mu \mathrm{m})$. Bioretention treatment for Sep runoff was bioretention soil media (BSM) overlying gravel. Leaching tests were clean embryo medium passed through BSM alone or BSM overlying gravel.

\begin{tabular}{|c|c|c|c|c|c|c|c|c|c|}
\hline \multirow[b]{2}{*}{ Parameter } & \multirow[b]{2}{*}{ Units } & \multicolumn{3}{|c|}{ Unfiltered Runoff } & \multicolumn{2}{|c|}{ Sep Bioretention } & \multicolumn{3}{|c|}{ Leaching Test } \\
\hline & & May $^{1}$ & Sep $^{1}$ & $\mathrm{Nov}^{2}$ & Plants $^{1}$ & $\begin{array}{c}\text { No } \\
\text { Plants }^{1}\end{array}$ & $\begin{array}{l}\text { Control } \\
\text { Water }\end{array}$ & $\begin{array}{c}\text { BSM } \\
\text { Alone }\end{array}$ & $\begin{array}{l}\text { BSM + } \\
\text { Gravel }\end{array}$ \\
\hline $\mathrm{ADP}^{\mathrm{a}}$ & Days & 15 & $50^{b}$ & 5 & n.a. ${ }^{c}$ & n.a. & n.a. & n.a. & n.a. \\
\hline $\mathrm{Zn}$ & $\mu \mathrm{g} / \mathrm{L}$ & 318 & 887 & n.m..$^{d}$ & 9 & 8 & 5 & 12 & 11 \\
\hline $\mathrm{Cu}$ & $\mu \mathrm{g} / \mathrm{L}$ & 251 & 102 & n.m. & 31 & 29 & 1 & 10 & 24 \\
\hline $\mathrm{Ni}$ & $\mu \mathrm{g} / \mathrm{L}$ & 14 & 7 & n.m. & 5 & 5 & $<0.5^{\mathrm{e}}$ & 9 & 9 \\
\hline $\mathrm{Pb}$ & $\mu \mathrm{g} / \mathrm{L}$ & 1.2 & 2.8 & n.m. & 0.33 & 0.25 & $<0.1$ & 2.6 & 2.6 \\
\hline $\mathrm{Cd}$ & $\mu \mathrm{g} / \mathrm{L}$ & 0.5 & 0.3 & n.m. & $<0.1$ & $<0.1$ & n.m. & n.m. & n.m. \\
\hline $\mathrm{pH}$ & std units & 7.0 & 7.0 & 7.8 & 6.6 & 6.7 & 7.4 & 7.1 & 7.3 \\
\hline Alkalinity & $\mathrm{mg} / \mathrm{L} \mathrm{CaCO}_{3}$ & 131 & 59 & n.m. & 105 & 115 & 4 & 25 & 22 \\
\hline $\mathrm{Ca}$ & $\mathrm{mg} / \mathrm{L}$ & 85 & 32 & n.m. & 43 & 51 & 9 & 35 & 36 \\
\hline $\mathrm{Mg}$ & $\mathrm{mg} / \mathrm{L}$ & 8 & 5 & n.m. & 8 & 10 & 26 & 13 & 11 \\
\hline Hardness & $\mathrm{mg} / \mathrm{L} \mathrm{CaCO}_{3}$ & 250 & 100 & n.m. & 140 & 167 & 130 & 140 & 130 \\
\hline $\begin{array}{l}\text { TSS }^{f} \\
\text { Total }\end{array}$ & $\mathrm{mg} / \mathrm{L}$ & 238 & 65 & n.m. & 37 & 25 & n.m. & n.m. & n.m. \\
\hline Ammonia $^{\mathrm{g}}$ & $\mathrm{mg} \mathrm{N} / \mathrm{L}$ & 5.7 & 3.0 & n.m. & 0.1 & 0.1 & n.m. & n.m. & n.m. \\
\hline TOC & $\mathrm{mg} / \mathrm{L}$ & 226 & 103 & n.m. & 62 & 60 & $<1.5$ & 16 & 12 \\
\hline DOC & $\mathrm{mg} / \mathrm{L}$ & 219 & 96 & n.m. & 58 & 58 & $<1.5$ & 16 & 12 \\
\hline TPAH $^{h}$ & $\mu \mathrm{g} / \mathrm{L}$ & 9.1 & 1.6 & n.m. & 0.1 & 0.1 & n.m. & n.m. & n.m. \\
\hline
\end{tabular}

${ }^{1}$ Collection and analytical chemistry methods described in Mclntyre et al. 2014. Sci. Tot. Environ. 500-501:173

${ }^{2}$ Collection methods described in Spromberg et al. 2015. J. Appl. Ecology. In Press.

a antecedent dry period

${ }^{b}$ runoff diluted $40 \%$ with roof-collected rain water

${ }^{\mathrm{c}}$ not applicable

${ }^{d}$ not measured

e below reporting limit

${ }^{f}$ total suspended solids

$\mathrm{g}$ Total ammonia nitrogen (sum of ionized and un-ionized by EPA Method 300.1)

${ }^{\mathrm{h}}$ total polycyclic aromatic hydrocarbons = sum of 19 parent and alkylated homologues 
Mclntyre et al. Molecular markers of stormwater runoff and bioretention treatment

Table S2. Individual PAHs $(\mu \mathrm{g} / \mathrm{L})$ measured in the May and September runoff as well as the September runoff following bioretention treatment.

\begin{tabular}{|c|c|c|c|c|}
\hline & & & Bioret & n-Treated \\
\hline Dissolved Parameter & May & Sep & Plants & No Plants \\
\hline Naphthalene & 0.2000 & 0.0370 & 0.0076 & 0.0085 \\
\hline C1-Naphthalene & 0.2300 & 0.0460 & 0.0082 & 0.0079 \\
\hline C2-Naphthalene & 0.1400 & 0.0400 & 0.0084 & 0.0072 \\
\hline C3-Naphthalene & 0.0930 & 0.0283 & 0.0061 & 0.0051 \\
\hline C4-Naphthalene & 0.1100 & 0.0193 & 0.0020 & 0.0015 \\
\hline Acenaphthylene & 0.0300 & 0.0047 & 0.0005 & 0.0012 \\
\hline Acenaphthene & 0.0110 & 0.0039 & $<0.001$ & $<0.001$ \\
\hline Fluorine & 0.0360 & 0.0094 & 0.0010 & 0.0018 \\
\hline C1-Fluorine & 0.0290 & 0.0058 & $<0.001$ & $<0.001$ \\
\hline C2-Fluorine & 0.1000 & 0.0177 & 0.0003 & $<0.001$ \\
\hline C3-Fluorine & 0.1900 & 0.0320 & $<0.001$ & $<0.001$ \\
\hline Dibenzothiophene & 0.0220 & 0.0062 & $<0.001$ & 0.0003 \\
\hline C1-Dibenzothiophene & 0.0400 & 0.0078 & $<0.001$ & $<0.001$ \\
\hline C2-Dibenzothiophene & 0.1300 & 0.0220 & 0.0006 & $<0.001$ \\
\hline C3-Dibenzothiophene & 0.2300 & 0.0347 & 0.0005 & $<0.001$ \\
\hline C4-Dibenzothiophene & 0.2300 & 0.0323 & $<0.001$ & $<0.001$ \\
\hline Phenanthrene & 0.3100 & 0.0803 & 0.009 & 0.016 \\
\hline C1-Phenanthrene & 0.3100 & 0.0553 & 0.004 & 0.005 \\
\hline C2-Phenanthrene & 0.4400 & 0.0743 & 0.004 & 0.003 \\
\hline C3-Phenanthrene & 0.4600 & 0.0817 & 0.002 & 0.002 \\
\hline C4-Phenanthrene & 0.3300 & 0.0627 & $<0.001$ & $<0.001$ \\
\hline Anthracene & 0.0280 & 0.0070 & 0.0015 & 0.0034 \\
\hline Pyrene & 0.4900 & 0.1013 & 0.0044 & 0.0054 \\
\hline Fluoranthene & 0.4000 & 0.1007 & 0.0045 & 0.0069 \\
\hline C1-Fluoranthene & 0.1500 & 0.0280 & 0.0010 & 0.0008 \\
\hline C2-Fluoranthene & 0.2600 & 0.0407 & 0.0015 & 0.0013 \\
\hline C3-Fluoranthene & 0.3500 & 0.0513 & 0.0010 & 0.0004 \\
\hline C4-Fluoranthene & 0.3500 & 0.0483 & $<0.001$ & $<0.001$ \\
\hline Chrysene & 0.3100 & 0.0510 & 0.0031 & 0.0021 \\
\hline C1-Chrysene & 0.3300 & 0.0513 & 0.0012 & 0.0012 \\
\hline C2-Chrysene & 0.5400 & 0.0793 & 0.0016 & 0.0015 \\
\hline C3-Chrysene & 0.5100 & 0.0740 & $<0.001$ & $<0.001$ \\
\hline C4-Chrysene & 0.4500 & 0.0673 & $<0.001$ & $<0.001$ \\
\hline Benzo[a]anthracene & 0.0840 & 0.0133 & 0.0009 & 0.0006 \\
\hline Benzo[b]fluoranthene & 0.1900 & 0.0263 & 0.0012 & 0.0011 \\
\hline Benzo[k]fluoranthene & 0.1300 & 0.0180 & 0.0009 & $<0.001$ \\
\hline Perylene & 0.2100 & 0.0317 & 0.0011 & 0.0010 \\
\hline Benzo[a]pyrene & 0.0930 & 0.0137 & 0.0004 & $<0.001$ \\
\hline Benzo[e]pyrene & 0.0410 & 0.0064 & 0.0008 & 0.0012 \\
\hline Indeno[1,2,3-cd]pyrene & 0.1400 & 0.0180 & 0.0007 & $<0.001$ \\
\hline $\operatorname{Dibenz}[a, h]$ anthracene (and $[a, c]$ ) & 0.0270 & 0.0034 & $<0.001$ & $<0.001$ \\
\hline Benzo[ghi]perylene & 0.3700 & 0.0507 & 0.0010 & 0.0007 \\
\hline
\end{tabular}


Table S3. Quantitative PCR (qPCR) primer information.

\begin{tabular}{|c|c|c|c|c|}
\hline Gene & $\begin{array}{l}\text { Primers: } \\
\text { Forward }\left(5^{\prime}-3^{\prime}\right) \text { Reverse }\left(3^{\prime}-5^{\prime}\right)\end{array}$ & $\begin{array}{l}\text { Amplicon } \\
\text { (bp) }\end{array}$ & NCBI Accession & $\begin{array}{l}\text { Published }^{\text {Primer }^{\mathrm{a}}}\end{array}$ \\
\hline $\begin{array}{l}a m h c \\
(m y h 6)\end{array}$ & $\begin{array}{l}\text { F: ATGACGTGTCAACACAGAAAGC } \\
\text { R: CTCAAGTTGCCTTCTCAGGTCT }\end{array}$ & 149 & NM_198823.1 & \\
\hline $\begin{array}{l}c m / c 1 \\
(m y / 6)\end{array}$ & $\begin{array}{l}\text { F: GGAAACGGAACAGTGATGGGTGCT } \\
\text { R: ACCCGGAGAGGATGTGCTTGATGA }\end{array}$ & 157 & NM_131692.1 & \\
\hline $\begin{array}{l}\mathrm{cmcl2} \\
(\mathrm{myl} / \mathrm{)})\end{array}$ & $\begin{array}{l}\text { F: AGGAGTTTAAGGAGGCTTTTGG } \\
\text { R: CCTTTTCCTTCTGTTAGCATGG }\end{array}$ & 145 & NM_131329.3 & \\
\hline cyp1a & $\begin{array}{l}\text { F: GGGAAAGAGTCCCAAATATTCC } \\
\text { R: CTCATATTAACCAGTCGCACCA }\end{array}$ & 216 & NM_131879.1 & \\
\hline$m t 2$ & $\begin{array}{l}\text { F: GCCAAGACTGGAACTTGCAAC } \\
\text { R: CGCAGCCAGAGGCACACT }\end{array}$ & 124 & NM_001131053.2 & 1 \\
\hline nppa & $\begin{array}{l}\text { F: GATGTACAAGCGCACACGTT } \\
\text { R: TCTGATGCCTCTTCTGTTGC }\end{array}$ & 110 & NM_198800.3 & 2 \\
\hline$n p p b$ & $\begin{array}{l}\text { F: CATGGGTGTTTTAAAGTTTCTCC } \\
\text { R: CTTCAATATTTGCCGCCTTTAC }\end{array}$ & 101 & XM_002663224.3 & 2,3 \\
\hline$t b \times 5 a$ & $\begin{array}{l}\text { F: CAGCGACTGGAGCCATAAGCTCAC } \\
\text { R: GGCCGGTAGTAGTGGTCATCCTCA }\end{array}$ & 217 & NM_130915.1 & \\
\hline $\begin{array}{l}v m h c \\
(m y h 7)\end{array}$ & $\begin{array}{l}\text { F: AATGAGACTGTGGTGGGACTCT } \\
\text { R: AGACACAGTCTGGAAGGAGGAG }\end{array}$ & 150 & NM_001112733.1 & \\
\hline$w d t c 1$ & $\begin{array}{l}\text { F: GCAGCGCTCTTCTCCAAAAC } \\
\text { R: CGACTCCTTCCGGCTGAAAT }\end{array}$ & 87 & NM_001130606.1 & \\
\hline
\end{tabular}

${ }^{\mathrm{a}}$ References for previously published qPCR primers

1. Chan, K. M.; Ku, L. L.; Chan, P. C. Y.; Cheuk, W. K., Metallothionein gene expression in zebrafish embryo-larvae and ZFL cell-line exposed to heavy metal ions. Mar. Environ. Res. 2006, 62, S83-S87.

2. Becker, J. R.; Robinson, T. Y.; Sachidanandan, C.; Kelly, A. E.; Coy, S.; Peterson, R. T.; MacRae, C. A., In vivo natriuretic peptide reporter assay identifies chemical modifiers of hypertrophic cardiomyopathy signalling. Cardiovasc. Res. 2012, 93, (3), 463-470.

3. Becker, J. R.; Chatterjee, S.; Robinson, T. Y.; Bennett, J. S.; Panakova, D.; Galindo, C. L.; Zhong, L.; Shin, J. T.; Coy, S. M.; Kelly, A. E.; Roden, D. M.; Lim, C. C.; MacRae, C. A., Differential activation of natriuretic peptide receptors modulates cardiomyocyte proliferation during development. Development 2014, 141, (2), 335-345. 
Mclntyre et al. Molecular markers of stormwater runoff and bioretention treatment

Table S4. Fold-changes in expression of molecular biomarkers of exposure and candidate biomarkers of cardiotoxicity relative to controls for embryonic zebrafish exposed to runoff events ranging in toxicity from strong (May), to moderate (Sep), to weak (Nov), with standard deviations in square brackets.

\begin{tabular}{|c|c|c|c|c|c|c|}
\hline Gene & Location $^{\mathrm{a}}$ & Role & May & Sep & Nov & $D^{6}$ \\
\hline \multirow[t]{2}{*}{ сур1a } & Whole body & Detox PAHs & $123.4^{*}$ & $36.4^{*}$ & $13.4^{*}$ & $\checkmark$ \\
\hline & & & [24.1] & [10.9] & [3.3] & \\
\hline \multirow[t]{2}{*}{$m t 2$} & Whole body & Detox metals & $4.2^{*}$ & $8.9 *$ & $2.0^{*}$ & \\
\hline & & & {$[1.5]$} & {$[6.2]$} & {$[0.6]$} & \\
\hline \multirow[t]{2}{*}{ nppa } & Heart & Contractility & $7.0^{*}$ & $7.4^{*}$ & 1.3 & \\
\hline & & & {$[2.3]$} & {$[2.7]$} & {$[0.6]$} & \\
\hline \multirow[t]{2}{*}{$n p p b$} & Heart & Contractility & $41.1 *$ & $6.1^{*}$ & 0.8 & $\checkmark$ \\
\hline & & & {$[7.4]$} & {$[1.6]$} & {$[0.4]$} & \\
\hline \multirow[t]{2}{*}{$c m / c 1(m y / 6)$} & Heart & Structural & $5.1^{*}$ & $3.1^{*}$ & 0.9 & $\checkmark$ \\
\hline & & & [0.9] & {$[0.6]$} & {$[0.7]$} & \\
\hline \multirow[t]{2}{*}{$\mathrm{cm} / \mathrm{c} 2(\mathrm{my} / 7)$} & Heart & Regulatory & $4.3^{*}$ & $2.9^{*}$ & 0.7 & $\checkmark$ \\
\hline & & & {$[0.3]$} & {$[0.9]$} & {$[0.2]$} & \\
\hline \multirow[t]{2}{*}{ amhc (myh6) } & Heart & Structural & 2.2 & $3.1^{*}$ & 0.9 & \\
\hline & & & [0.7] & [1.3] & {$[0.2]$} & \\
\hline \multirow[t]{2}{*}{ vmhc (myh7) } & Heart & Structural & 1.1 & 1.4 & 0.7 & \\
\hline & & & [0.2] & {$[0.2]$} & {$[0.2]$} & \\
\hline \multirow[t]{2}{*}{$t b \times 5 a$} & Heart, eye & Transcription factor & 1.1 & $2.5^{*}$ & 0.9 & \\
\hline & & & {$[0.2]$} & [1.1] & {$[0.1]$} & \\
\hline \multirow[t]{2}{*}{$w d t c 1$} & Heart & Reference & 1.0 & 0.5 & 1.2 & \\
\hline & & & {$[0.2]$} & {$[0.2]$} & {$[0.4]$} & \\
\hline
\end{tabular}

${ }^{a}$ Tissue expression: ZFIN Database, embryo-larval stage zebrafish (www.zfin.org)

${ }^{\mathrm{b}}$ correlated with cardiac toxicity across treatments

* significant changes in gene expression, $\mathrm{p}<0.05$ 
Mclntyre et al. Molecular markers of stormwater runoff and bioretention treatment

Table S5. Expression of genes either significantly affected by filtered runoff relative to control water $(*)$ or not significantly different than unfiltered runoff or control water. Standard deviation of fold-change in square brackets.

\begin{tabular}{llcccc} 
& & \multicolumn{3}{c}{ Filtered Runoff } & \multicolumn{2}{c}{ Control Water } \\
\hline Gene & Tissue & Plants $^{\mathrm{a}}$ & No Plants $^{\mathrm{b}}$ & BSM $^{\mathrm{c}}$ only & BSM + gravel \\
\hline cyp1a & Whole body & $6.2^{*}$ & $4.8^{*}$ & 0.8 & 0.7 \\
& & {$[3.8]$} & {$[3.1]$} & {$[0.6]$} & {$[0.3]$} \\
nppa & Heart & $4.7^{*}$ & 3.3 & $1.6^{*}$ & 1.5 \\
& & {$[2.7]$} & {$[2.7]$} & {$[0.2]$} & {$[0.4]$}
\end{tabular}

${ }^{\mathrm{a}}$ bioretention soil media (BSM; treatment) including plants

${ }^{\mathrm{b}}$ bioretention soil media (treatment) without plants

${ }^{\mathrm{c}}$ bioretention soil media 



Figure S1. Cardiac-related morphometrics and transcriptional response of $n p p b$ to dilutions of the unfiltered May and September runoff events in zebrafish embryos following a $48 \mathrm{~h}$ exposure. Pericardial area (PCA) is included within the periventral area (PVA). PVA is referred to in the text as the area of the common cardinal vein. 




Figure S2. Immunofluorescence in zebrafish exposed to control water, unfiltered runoff, or runoff filtered with plants or without plants. Red staining (MF20) marks myosin heavy chain of the heart and skeletal muscles. Green staining (C10-70) marks CYP1A protein expression. Fish are positioned anterior to the left except for ventral view, which is anterior up (up and left for Plants and No Plants). In cranial views, embryo is rotated slightly to dorsal view. Visible stained muscles (red) include the adductor mandibulae (am) and hyohyoideus (h) in the head, the myocardium (mc), pectoral fin (pec), and the somites (so) of the trunk. In unfiltered runoff, CYP1A is present throughout the epidermis and the vasculature, including the endocardium. In filtered runoff, epithelial and endocardial staining for CYP1A are no longer present, but mild staining persists in the peripheral vascular. 